\title{
Une double sélection, positive et négative, des thymocytes explique la tolérance immunitaire et la spécificité restreinte dans le système majeur d'histocompatibilité
}

Il est généralement admis que les lymphocytes $\mathrm{T}$ exprimant les molécules CD4 et CD8 $\left(\mathrm{CD}^{+}{ }^{+}, \mathrm{CD}^{+}\right)$sont les précurseurs des lymphocytes matures $\mathrm{CD}^{+}{ }^{+} \mathrm{CD}^{-}$et $\mathrm{CD}^{-}, \mathrm{CD}^{+}$ qui composent, respectivement, les cellules auxiliaires et les cellules cytotoxiques (voir lexique $\mathrm{m} / \mathrm{s} n^{\circ} 4$, vol. 3, p. 229). L'une et l'autre de ces populations reconnaissent les antigènes étrangers présentés par des molécules du CMH (complexe majeur d'histocompatibilité) identiques au leur propre (phénomène de spécificité restreinte), mais ni les auto-antigènes ni les antigènes étrangers présentés par d'autres molécules du CMH. L'absence de cellules autoréactives est habituellement attribuée à un phénomène de destruction des clones dont le récepteur reconnaît un antigène du self (auto-antigène). La restriction dans le système d'histocompatibilité (c'est-à-dire la reconnaissance des antigènes étrangers uniquement dans le contexte des molécules du $\mathrm{CMH}$ similaires à celles des lymphocytes $T$ ) suggère qu'il pourrait exister, de plus, une sélection positive des clones de cellules $\mathrm{T}$ immatures dont le récepteur pour l'antigène reconnaît les molécules du CMH homologues. Plusieurs expériences utilisant des souris transgéniques sont venues récemment à l'appui de cette théorie [1-3]. Des souris transgéniques qui expriment les gènes $\alpha$ et $\beta$ codant pour les deux chaînes d'un récepteur $T$ spécifique d'antigènes présentés dans le contexte de molécules du $\mathrm{CMH}$ de classe I ont été créées. La plupart des lymphocytes de tels animaux expriment le transgène et non les gènes

récepteur car, comme dans le cas des immunoglobulines, l'expression d'un gène réarrangé (ici le transgène) inhibe le réarrangement ultérieur des autres gènes homologues, non encore réarrangés. Lorsque les souris possèdent les molécules du $\mathrm{CMH}$ reconnues par le récepteur transgénique, les lymphocytes matures sont déplétés en cellules $\mathrm{CD}^{+}{ }^{+}$et presque toutes les cellules exprimant le transgène sont $\mathrm{CD}^{-}, \mathrm{CD}^{+}$, ce qui s'explique si on se rappelle que CD4 interagit spécifiquement avec les molécules du CMH de classe II et CD8 avec celles de classe I : ce sont des lymphocytes $\mathrm{CD}^{+}$qui peuvent reconnaître les cibles présentées au récepteur transgénique dans le contexte des molécules de classe I. $\mathrm{Si}$, de plus, la souris transgénique exprime l'antigène reconnu par le récepteur $T$, il y a disparition complète des cellules $\mathrm{T}$ autoréactives, c'est-à-dire des lymphocytes $\mathrm{CD}^{+}$ exprimant le transgène. Si, enfin, le transgène est exprimé dans une souris de CHM différent de celui reconnu par le récepteur, il n'y a ni sélection ni délétion de clones. L'ensemble de ces résultats, particulièrement bien étudiés par les équipes de l'institut d'immunologie et du centre Hoffman-Laroche de Bâle (Suisse) [2], est parfaitement cohérent avec l'hypothèse d'une sélection positive et d'une sélection négative. La sélection négative exige la reconnaissance par la cellule T de l'autoantigène dans le contexte du $\mathrm{CMH}$ homologue et la sélection positive la reconnaissance du $\mathrm{CMH}$ homologue sans l'auto-antigène... ce qui soulève une question: sous quelle forme se trouvent les molécules du $\mathrm{CMH}$ assurant la sélection positive? Sontelles «vides», ne présentant aucun antigène (ce qui n'est peut-être pas possible, voir $\mathrm{m} / \mathrm{s} n^{\circ} 1$, vol. 4 , p. 52), ou bien présentent-elles des antigènes du self différents de ceux assurant la déplétion clonale des cellules autoréactives ?

Il existe des arguments pour penser que la sélection positive se passe dans le cortex thymique et précède la sélection négative qui se déroulerait dans la médullaire du même organe. Les auto-antigènes du cortex thymique pourraient par conséquent avoir une structure et une signification différentes de celles des antigènes de la médullaire qui seraient, eux, les auto-antigènes «classiques » contre lesquels s'établit la tolérance immunitaire. Alternativement, la sélection positive pourrait n'intéresser que les clones exprimant un récepteur $\mathrm{T}$ de faible affinité pour les molécules du $\mathrm{CMH}$ homologue, qu'ils soient «vides» ou qu'ils présentent un antigène, quelconque. Parmi ces clones sélectionnés positivement (et par conséquent spécifiques du $\mathrm{CMH}$ homologue), ceux se liant avec une forte affinité à des molécules du $\mathrm{CMH}$ présentant un antigène du self seraient éliminés dans le thymus au cours du développement. Ceux reconnaissant, plus tard, un antigène étranger, seraient stimulés à se diviser, donnant le phénomène de réponse immunitaire «restreinte» dans le système du CMH (figure 1). Il semble que les molécules du $\mathrm{CMH}$ de classe II et CD4 soient impliqués dans le phénomène de délétion des clones autoréactifs. Celle-ci n'a en effet pas lieu lorsque l'on injecte dans l'animal nouveau-né des anti- 


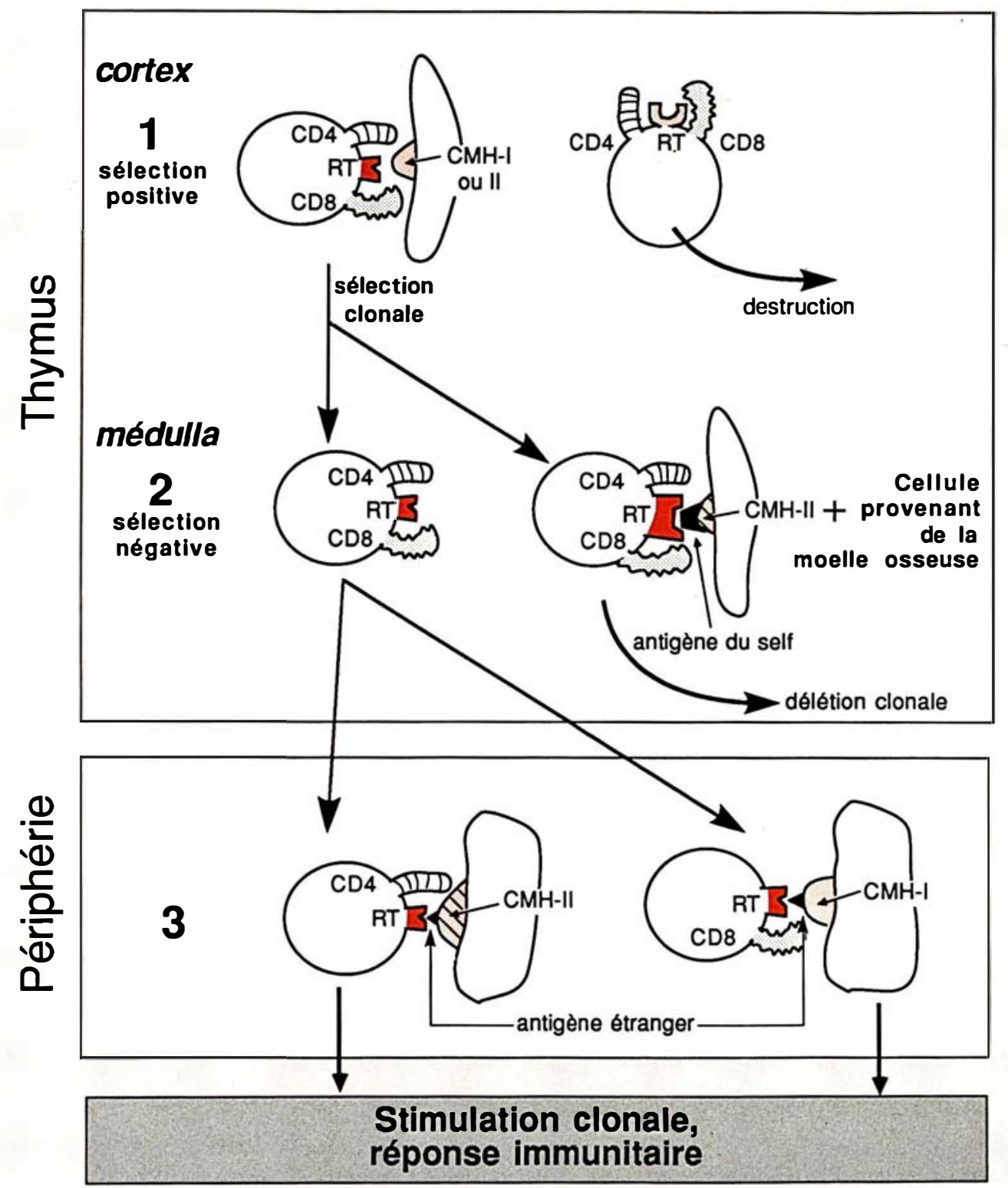

Figure 1. Modèle possible de la sélection positive et négative des thymocytes au cours de leur maturation. II faut bien noter que le schéma présenté ci-dessus n'est qu'un des modèles possibles et que beaucoup de points restent incertains et spéculatifs. Étape 1, se déroulant dans le cortex thymique. Seuls survivent les lymphocytes $\mathrm{CD4^{+ }}$, CD8 ${ }^{+}$qui reconnaissent des molécules du CMH homologue (faiblement... ou lorsqu'elles présentent des antigènes du self particuliers, uniquement présents dans le cortex), les autres étant éliminés. Cette sélection est à la base de la spécificité restreinte, puisque tous les clones survivants ne pourront reconnaître un antigène que dans le contexte des molécules du CMH homologue. II est possible que les précurseurs sélectionnés par des molécules du CMH de classe I soient prédéterminés pour donner les cellules matures $C D 4^{-}, C D 8^{+}$alors que les précurseurs sélectionnés par des molécules de classe ll seraient à l'origine des populations matures $\mathrm{CD}^{+}, \mathrm{CD} 8^{-}$. Étape 2. Parmi les clones sélectionnés pour leur aptitude à reconnaître les molécules du CMH homologue, ceux reconnaissant des antigènes du self présentés par des molécules de classe ll seraient éliminés, évitant ainsi l'existence de clones autoréactifs. Cette étape de délétion clonale se ferait dans la médullaire thymique, en présence de cellules provenant de la moelle osseuse. Étape 3. Les précurseurs ayant subi la double sélection se différencient en les deux populations matures $\mathrm{CD}^{+}, \mathrm{CD} 8^{-}$et $\mathrm{CD4}^{-}, \mathrm{CD} 8^{+}$. La prolifération des clones reconnaissant spécifiquement un antigène étranger présenté par les molécules du CMH homologue est stimulée, expliquant la réponse immunitaire à la stimulation antigénique.

$\mathrm{m} / \mathrm{s} n^{\circ} 10$ vol. 4, décembre 88 
médecine/sciences $1988 ; 4: 658-9$

corps anti-CD4. Ceux-ci bloquent la sélection positive et on ne retrouve pas de clones $\mathrm{CD}^{+}, \mathrm{CD}^{-}$; ils bloquent également la sélection négative... y compris des clones CD4$\mathrm{CD}^{+}[4,5]$.

Les expériences de Kisielow et al. [6] indiquent enfin que la sélection (au moins la sélection négative, mais aussi, évidemment, la sélection positive si elle est antérieure) se fait au niveau des précurseurs $\mathrm{CD} 4^{+}, \mathrm{CD}^{+}$: des souris transgéniques pour les gènes d'un récepteur $\alpha \beta$ spécifique de l'antigène HY (exprimé chez les mâles) présenté dans le contexte de molécules du $\mathrm{CMH}$ de classe I ne possèdent, chez les animaux mâles ayant les molécules du CMH pour lesquelles est restreint le récepteur transgénique, ni les cellules autoréactives matures $\mathrm{CD}^{-} \mathrm{CD}^{+}$ni les cellules précurseurs $\mathrm{CD}^{+} \mathrm{CD}^{+}$, alors que, chez les femelles (n'exprimant pas HY), ces cellules immatures $\mathrm{CD}^{+} \mathrm{CD}^{+}$exprimant le transgène sont nombreuses. Ainsi, pas à pas, grâce à l'éventail entier des techniques de recombinants d'ADN et d'immunologie, élucide-t-on en cette deuxième moitié des années 80 les bases moléculaires du système immunitaire et de son aptitude à différencier le soi du non-soi [7].

A. $\mathbf{K}$.

1. Hung Sia Teh, Kisielow P, Scott B, et al. Thymic major histocompatibility complex antigens and the $\alpha \beta$ T-cell receptor determine the CD4/CD8 phenotype of T cells. Nature $1988 ; 335: 229-33$.

2. Sha WC, Nelson CA, Newberry RD, Kranz DM, Russel JH, Loh DY. Selective expression of an antigen receptor on CD8bearing $T$ lymphocytes in transgenic mice. Nature 1988; 355 : 271-4.

3. Janeway CA. T-cell development : accessories or coreceptors. Nature 1988 ; 335 : 208-10. 4. Fowlkes BJ, Schwartz PH, Pardoll DM. Deletion of self-reactive thymocytes accurs at a CD4 ${ }^{+} \mathrm{CD}^{+}$precursor stage. Nature 1988 ; 334: 620-3.

5. MacDonald HP, Hengartner H, Pedrazzini $T$. Intrathymic deletion of self-reactive cells prevented by neonatal anti-CD4 antibody treatment. Nature 1988 ; 335 : 174-6.

6. Kisielow $\mathrm{P}, \mathrm{Blüthmann} \mathrm{H}$, Staerz VD, et al. Tolerance in T-cell-receptor transgenic mice involves deletion of nonmature $\mathrm{CD}^{+}, \mathrm{CD}^{+}$ thymocytes. Nature $1988 ; 333: 742$

7. Kourilsky P, Claverie J-M. Le modèle du soi peptidique. médecine/sciences $1988 ; 4$ :

\section{Des modèles animaux pour l'étude du SIDA}

L'absence de modèle animal d'une maladie, notamment d'une maladie infectieuse, constitue un considérable handicap pour l'étude physiopathologique de l'affection et pour les essais thérapeutiques. Les chimpanzés sont sensibles à l'infection par les virus du SIDA, mais développent une maladie inapparente. De plus, ils sont rares, chers, et leur espèce est considérée comme menacée de disparition. De nombreuses tentatives ont donc été entreprises de développer un modèle animal plus accessible.

L'une des tentatives les plus folles, voire les plus inquiétantes, a été récemment rapportée par l'équipe de Frederickson (NCI, Bethesda MD, USA) lors d'un récent colloque du Cold Spring Harbor laboratory, près de New York. Il s'agit de créer des animaux transgéniques ayant intégré la totalité du génome viral de HIV-l. Les souris ainsi produites développent au $10^{\mathrm{e}}$ jour de vie une maladie caractérisée par un retard de croissance, une infiltration lymphoïde périvasculaire dans le poumon, des signes cutanés évoquant un psoriasis, des adénopathies et une réponse anormale des lymphocytes $T$ aux mitogènes. Du virus infectieux est retrouvé dans la rate et les ganglions. Les animaux meurent aux environs du $30^{\mathrm{e}}$ jour. Les souris ayant une tendance difficilement contrariable à s'échapper, ce sont de véritables quartiers de haute sécurité comportant trois lignes indépendantes de protection contre la fuite des animaux qui ont dû être construits, afin d'éviter la dissémination de ces petites bêtes produisant du virus HIV!

D'autres animaux transgéniques exprimant la molécule humaine CD4, récepteur des virus HIV, ont été créés dans l'espoir - déçu pour l'instant - que cela suffirait à permettre d'infecter ces animaux par HIV. En fait, le virus est bien intégré dans les cellules de souris, mais il ne s'y réplique pas, ce qui semble indiquer qu'existe une barrière d'espèce intracellulaire au cycle viral de HIV.

Très récemment a été développé un modèle tout différent et qui pourrait être promis à un avenir extrêmement important : il s'agit de construire des chimères de souris possédant de manière stable les cellules immunitaires de l'espèce humaine. Ceci est envisageable grâce à la mutation SCID (severe combined immunodeficiency). Les souris homozygotes pour l'allèle autosomique scid, d'apparition spontanée, ont un déficit immunitaire touchant les cellules $B$ et les cellules $T$. L'anomalie ne porte pas sur les autres lignées hématopoïétiques ; elle semble intéresser, spécifiquement, le processus de recombinaison des segments constituant les gènes d'immunoglobuline et des récepteurs pour l'antigène des lymphocytes T. Ce serait le système de la «recombinase» qui serait anormal : capable d'entraîner la coupure de l'ADN au niveau des sites corrects, il ne catalyserait pas la réunion normale des segments, ce qui provoque des délétions de régions entières des gènes d'immunoglobuline et des récepteurs $T$. Du fait du déficit immunitaire, les souris scid/scid ne rejettent pas les xénogreffes (greffes en provenance d'autres espèces). D'autre part, des cellules du système immunitaire humain fœtal greffées à ces souris développent une tolérance immunitaire vis-à-vis des tissus de souris, si bien qu'il n'y a pas non plus de réaction du greffon contre l'hôte[l, 2]. Lorsque du thymus, du tissu ganglionnaire et du foie foetaux sont greffés à ces souris, on observe l'apparition de cellules $\mathrm{T}$ et d'anticorps humains durant plusieurs semaines (7 semaines en moyenne, 